\title{
La competitividad de las PYMES del sector calzado y su eficiencia en la satisfacción de las necesidades del consumidor
}

\section{The competitiveness of SMEs in the footwear sector and their efficiency in satisfying consumer needs}

\author{
MBA. Leonardo Ballesteros-López \\ leonardogballesteros@uta.edu.ec \\ Mg. Santiago Peñaherrera-Zambrano ${ }^{2}$ \\ spenaherrera@uta.edu.ec \\ Mg. César Guerrero-Velástegui ${ }^{3}$ \\ ca.guerrero@uta.edu.ec \\ Ing. Carlos Masaquiza-Caiza \\ cmasaquiza@bhconsultores.com
}

Recibido: 1/09/2017, Aceptado: 1/11/2017

\begin{abstract}
RESUMEN
El presente trabajo se fundamenta en el estudio de la competitividad que existe en la producción de calzado de las PYMES del sector calzado en la parroquia de Ambatillo. Partiendo de la premisa que las empresas actuales deben apuntar a la productividad, competitividad e innovación para ser parte activa del mundo globalizado, logrando una ventaja competitiva para así sobresalir de las demás ya que en el sector hemos encontrado una muestra de 55 PYMES, razón por la cual se escogió esta parroquia para la realización de este estudio, las empresas de este sector afirman que su producción es realizada con materia prima de alta calidad rigiéndose a las normas establecidas a la ley con una adaptación constante a los cambios y crisis ocurridas en el país, es por eso que se realizó una investigación para determinar aspectos organizacionales, disposiciones gerenciales y las distintas formas de competitividad a las que estas se pueden enfrentar realizando encuestas de las cuales podremos observar los canales de distribución utilizados, el porcentaje de ventas, tipo de empresa, calidad del producto, promoción del producto, adaptación a cambios, calidad de la materia prima utilizada en las PYMES encuestadas.
\end{abstract}

Palabras clave: Competitividad, ventaja competitiva, innovación, desarrollo, calzado, PYMES, producción

\footnotetext{
1 Docente de la Universidad Técnica de Ambato. Ecuador

2 Docente de la Universidad Técnica de Ambato. Ecuador

3 Docente de la Universidad Técnica de Ambato. Ecuador

4 BH Consultores. Ecuador
} 


\begin{abstract}
The present article is based on the study of the competitiveness that exists in the production of footwear of the SMEs of the footwear sector in the parish of Ambatillo. Starting from the premise that today's companies must aim at productivity, competitiveness and innovation to be an active part of the globalized world, achieving a competitive advantage to excel in the others since in the sector we have found a sample of 55 SMEs, Which chose this parish for the realization of this study, the companies of this sector affirm that their production is made with high quality raw material, following the norms established to the law with a constant adaptation to the changes and crises that occurred in the Country, that is why an investigation was carried out to determine organizational aspects, managerial dispositions and the different forms of competitiveness that can be faced by conducting surveys of which we can observe the distribution channels used, the percentage of sales, type of Company, product quality, promotion of the product, adaptation to changes, quality of the raw material used in the SMEs surveyed.
\end{abstract}

Keywords: Competitiveness, competitive advantage, innovation, development, footwear, SMEs, production

\title{
Introducción
}

Las pymes llegan a replantar un sector empresarial muy importante dentro de la economía en la cual, la alta competitividad de las pequeñas y medianas empresas (PYMES) esta enfocadas con una oferta sus productos las cuales intentan cubrir un numero de necesidades de esta manera la competitividad que existe dentro de las mismas se basa en las estrategias que se utilice en el momento de la comercialización de sus productos las cuales tratan de evolucionar mediante los cambios que se dan en su entorno y de la misma manera los cuales les ayuda a ser más competitivos frente a la competencia y logrando así posición en el mercado.

La ventaja competitiva como un reto importante para las PYMES en la industria de calzado, adoptan reformas económicas que trajeron cambios drásticos en forma en que opera la economía de algunas empresas de esta manera tuvo por objeto la apertura de los mercados dejando a las pequeñas empresas como el reto de enfrentar a la competencia proveniente del exterior y de esta manera a la intemperie para operar sus negocios eficientemente. Debido al control gubernamental sobre diversas actividades las empresas e industrias de uso intensivo de mano de obra se vieron en la gran necesidad de adaptarse a los cambios drásticos de los países de bajos costos.

De esta manera, la industria de calzado tuvo una restructuración significativa internacional mente esto se debió a que muchos de los países se adaptaron y modificaron a la base competitiva de los cambios industriales. Debido a esto las pymes se vieron expuestas a la competencia internacional, tenían que desarrollar ventajas competitivas para así mantenerse en el mercado adaptándose de esta manera a los nuevos cambios industriales, ante estos hechos muchas pymes no sobrevivieron, pero de la misma manera otras empezaron a obtener una participación evidente (Stoney, Bowen, \& Stoney, 2016). Las estrategias de las Pymes pueden representar un esfuerzo de adaptación que se presenta en los cambios en el ambiente 
(Knight, 2000). La capacidad de adaptabilidad de una empresa está se la determina por su dinámica que está basado en el aprendizaje y experiencias ganadas con el tiempo de forma empírica (Palmer, Montalvo, \& Pletea, 2010), además, las pymes como estrategia pueden ser el resultado de un artículo deliberado, o de una confusión por accidentes, intrusiones o inercia del planteamiento de las mismas (Apps, Sterzing, Brien, \& Lake, 2017).

\section{Desarrollo}

La ventaja competitiva es un proceso dinámico esto se debe a los cambios que se producen en los diferentes sectores como el ambiente, los cambios de instituciones, organizaciones y empresas, los cambios deben ser adaptados a las necesidades del entorno como la adquisición de nuevos aprendizajes, cooperación, integración, todo lo que se refiera al apoyo y ayuda de las PYMES (Mu, García-Pinillos, \& Soto-Hermoso, 2016) Para que Las PYMES en el sector calzado sean más competitivas, puedan tomas decisiones apropiadas deben adaptarse y estar preparadas para los cambios en el mismo para así poder mantenerse en competencia (Esteban \& Cohen, 2013).

Las ventajas competitivas basados en los costos de (MO) Mano de Obra descarta otros aspectos que dan ventajas a las PYMES, para llevar acabo las ventajas competitivas las pequeñas empresas dependen principal mente de los medios con los que dispones ya sea que estos se hallen en la misma empresa y se las practique como las de innovación capacitación etc.

Después de considerar tanto tiempo a las PYMES como algo obsoleto se empieza a reconocer su importancia para el desarrollo económico y social, este tipo de empresas han tenido un gran desarrollo además de ser creadoras de valor agregado y fuentes de empleo una de las razones más importante por la que se ha dado el crecimiento de la PYMES es la dificultad económica siendo la está la principal generadora de empleo. Ambatillo es un sector altamente competitivo en la producción de calzado, y nos podemos encontrar con compradores que cada vez exigen mejoras en la calidad de la producción, así como en su precio y servicio, es por eso que es necesario desarrollar ventajas competitivas como una herramienta estratégica para lograr una alta posición en el mercado.

Las ventajas competitivas son un proceso dinámico ya que se enfrentan a distintos cambios además que es necesaria la adaptación a los distintos tipos de cambio que día a día acurren en la sociedad. Es por eso que es necesario un monitoreo de control y aprendizaje continuo para ayudar a las pymes a cumplir su objetivo.

Por lo general este tipo de empresas son familiares que iniciaron con la creación de talleres y poco a poco van creciendo adaptándose a distintos diseños internacionales o innovaciones propias que les ayude a sobresalir de la competencia además acuden a ferias de calzado donde pueden conocer nuevas tendencias moda y materia prima.

Al enfrentarse a tanta competencia en este sector este tipo de empresas han visto la necesidad de contratar personal especializado en el sector además de adquirir distintos sistemas que mejoren su logística y calidad (Marco \& Merlo, 2016). 


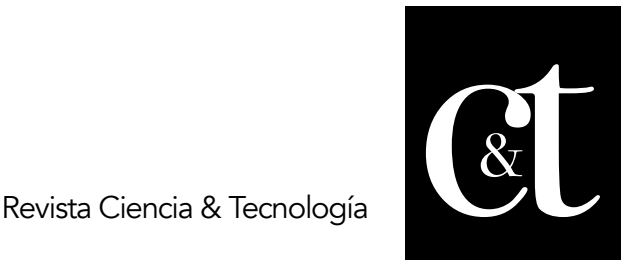

No. 17, 31 de enero de 2018

ISSN impreso: 1390 - 6321

Las financiaciones de las PYMES, los negocios frecuentemente inician con éxito tomando como base los ahorros personales o con dinero procedente de créditos obtenidos combase a una póliza de seguros para que puedan sobrevivir (Davis, Galna, Murphy, Williams, \& Haines, 2016).

Las PYMES se caracterizan por sus expectativas, podemos identificar sus problemas y a la vez saber cómo aplicar metodologías para poder resolverlos. Crecimiento: Influye en si a las actividades financieras y técnicas; es decir en el apoyo de la planeación y organización. Actividades sobresalientes: Es importante mencionar todas sus estructuras que va a fortalecer a las pymes. Las PYMES tienen varios beneficios para radicar en las industrias en particular a los individuos y a los países con quien se relacionan directamente. Las PYMES son estudiadas y analizadas muy a fondo por el ambiente externo mediante el cual son apoyadas por las habilidades internas de administración y así lograr una integración de factores (Luigi \& Antonio, 2016).

Los fracasos de los negocios ocurren porque la administración no pudo hacer frente a la elevación de los costos que resulta de la competencia; ya que es sorprendente como la competencia reduce sus costos y tiende a realizar una planificación obligada (Dickson, 1974). Una pequeña empresa es considerada como aquella que se desenvuelve en el ámbito local ya que es administrada por una o un pequeño grupo de personas estableciendo una comparación con otras empresas del mismo ramo; algunas de las ventajas de la pequeña empresa depende de su tamaño y de su gestión autónoma: por lo general en este tipo de empresa el manejo es único ya que existe un contacto directo entre jefe y empleados ya que esta relación ayuda a determinar varias posibilidades en el establecimiento como reducir muchos de los aspectos y problemas que se pueden suscitar en las grandes empresas, uno de los incentivos que tiene por ofrecer la pequeña empresa es que el propietario es quien lo maneja es decir trabaja por su propia cuenta y las ganancias que se obtengan son la retribución financiera del mismo (Hernández-Girón, Geleijns, Joemai, \& Veldkamp, 2015).

Los administradores tienden cada vez más a mejorar la producción ya que varía según las empresas al igual que los costos de producción (Juarez, 1986).

La mediana y pequeña empresa son una de las principales fuentes de economía del país ya que uno de sus principales motores es la cadena de produccióncomercialización por lo cual conlleva a generar puestos de trabajos directos o indirectos. Los factores que se inciden en la empresa se han ido modificando mediante la evolución de la tecnología del mundo actual, hoy en día la competencia obliga a aprovechar al máximo sus ganancias e inversiones porque las compañías grandes están bien preparadas para el mejoramiento de su supervivencia en general. Los éxitos de las medianas y pequeñas empresas se debe a que se obtienen muy pocos tropiezos ya que los minoristas conocen muy a fondo el negocio ya que no carecen de una visión global que tiene por objeto hacer funcionar la empresa bajo un punto de vista técnico es decir fabricar el producto (Irzmanska, 2016). 


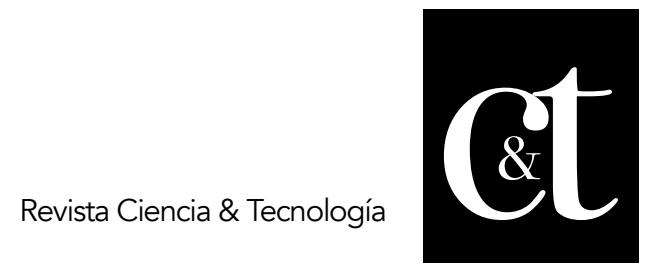

No. 17, 31 de enero de 2018

ISSN impreso: 1390 - 6321

Una empresa es una organización de individuos que trabajan por concluir con un bien común es decir un sustento económico ya que fundamentalmente es una asociación donde cada uno de las personas tienen distintos fundamentos ya sea social, cultural o psicológicos, las relaciones en el mundo de los negocios no fueron muy específicas porque existe una función denominada caja negra a la que se le inyecta componentes y se obtiene productos ya elaborados. La obsesión por el crecimiento de la empresa es un problema que ocasiona disturbios al empresario y puede provocar daños irreparables en la empresa; las PYMES constituyen en la empresa como un socio que ayuda a establecer directamente el punto de diferenciación de separar a las pequeñas y medianas empresas.

Las PYMES son el choque de las ideas dadas por el creador de la empresa y sus clientes. En las prácticas existe una variedad de actividades que por su complejidad no resulta conveniente que se tomen a su cargo (Karimi, Allahyari, Reza, \& Khalkhali, 2016). Las PYMES tienen algunas debilidades como la tasa alta de mortalidad ya que no tienen aprobada una idea nueva.

En las empresas existen las ventajas competitivas como el resultado de la adaptabilidad del ambiente en el que se encuentra, en un ambiente dinámico, estratégico, lógico y adaptable para las operaciones de las pequeñas empresas en la cual se presenta las ventajas competitivas (Area, 2013).

Las ventajas competitivas logran crear en los clientes una manera global de participación como estrategia de estabilidad que se adapta a las necesidades del entorno (Urriolagoitia \& Coronado, 2010).

Una microempresa tiene un mercado netamente local y/o regional es por eso que su crecimiento va encaminado ese tipo de mercados ya que no cuentan con el financiamiento suficiente lo que no les permite hacer cambios significativos para su crecimiento, y observando esta necesidad es como se van creando distintos organismos de apoyo financiero para los pequeños empresarios (Anzola, 2015).

El problema encontrado acerca de la competencia nos da como necesidad realizar un análisis de este el cual empieza identificando a los competidores potenciales de dos formas diferentes la primera eligiendo un grupo de compradores y analizar el nivel de elección del comprador; y la segunda agrupamos a los compradores y analizamos sus fortalezas y debilidades a la hora de realizar sus compras (Ortiz-Triana \& CaicedoRolón, 2014).

En los últimos años las PYMES han sufrido fuertes cambios tanto dentro como fuera del país, hoy en día las ventajas competitivas desarrolladas que aseguran la supervivencia de este tipo de empresas dependen cada día más de la tecnología es así como se tiene que ir desarrollando ventajas tecnológicas de producción para su crecimiento y desarrollo (Rengifo, Mena, Ramírez, \& Montoya, 2013).

\section{Metodología}

Es la descripción y argumentación de las principales decisiones metodológicas que se ven según los temas a investigar y las posibilidades del investigador acerca de sus 
características, cualidades que dan enfoque a las estructuras metodológicas para asegurar la validez de la investigación y su análisis crítico (Romero Sigüenza, 2015).

En este proyecto de investigación hemos aplicado los niveles de metodología de investigación a mencionarse:

Investigación descriptiva, en este nivel de investigación se describe las situaciones y eventos que se podrían manifestar dándonos a conocer las características que sobresalen en el problema de la competitividad de las PYMES de calzado que se encuentran en la parroquia de Ambatillo.

Investigación exploratoria, debido a la competitividad de las PYMES en el sector Ambatillo que se da en la producción de calzado como problema de investigación determinaremos el nivel de su competencia, analizando las principales causas de competitividad (Pacheco-Blanco, Collado-Ruiz, \& Capuz-Rizo, 2015).

Utilizaremos la investigación de campo ya que acudiremos a los puntos estratégicos donde se encuentran las PYMES para analizar la realidad de sus problemas, determinando por qué se produce una alta competencia de producción de calzado en este sector.

Aplicamos una investigación bibliográfica ya que analizamos distintos casos referentes al problema que se está desarrollando en la parroquia de Ambatillo en distintas fuentes como son libros, revistas, artículos científicos, tesis, y documentos relacionados al tema.

Aplicando las fórmulas de población que corresponde a las empresas productoras de calzado en la parroquia Ambatillo del Cantón Ambato, en la cual se tomó como referencia la población del sector correspondiente a PYMES de acuerdo a los datos de último del censo en la parroquia Ambatillo INEC 2010 que es de 100.

\section{Resultados y discusión}

A continuación, se reflejan los resultados principales que se presentaron en el transcurso de la investigación de acuerdo a las preguntas que se desarrolló en la encuesta dirigida a los productores de calzado de la parroquia Ambatillo, detallando:

- Canales de distribución

- Porcentaje de ventas de los productores

- Tipo de empresa

- Calidad de productos

- Promoción del producto

- Adaptación a cambios

- Calidad de la materia prima 
Tabla 1. Canales de Distribución utilizados

\begin{tabular}{|l|l|}
\hline PREGUNTA & PERSONAS \\
\hline Directos & 30 \\
\hline Indirectos & 25 \\
\hline TOTAL & 55 \\
\hline
\end{tabular}

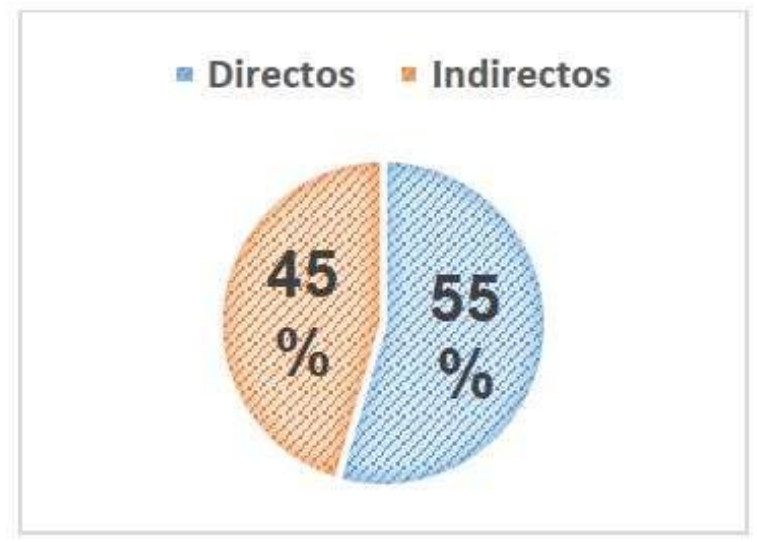

Gráfico 1. Canales de Distribución

La mayor parte de PYMES utilizan canales de distribución directa para la comercialización de sus productos, para así obtener mayores utilidades al dejar de utilizar intermediarios. 
Tabla 2. Porcentaje de Ventas

\begin{tabular}{|l|l|}
\hline PREGUNTA & PERSONAS \\
\hline Siempre & 29 \\
\hline A veces & 16 \\
\hline Nunca & 10 \\
\hline TOTAL & 55 \\
\hline
\end{tabular}

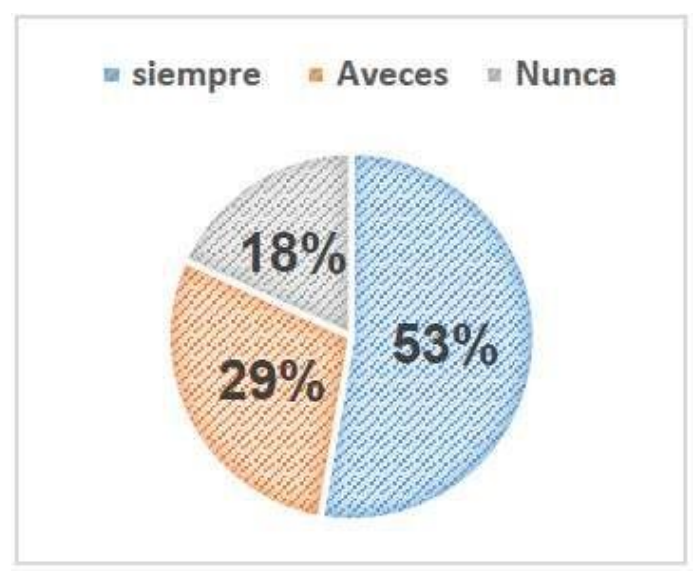

Gráfico 2. Porcentaje de ventas

La mayoría de las empresas establecidas en sector consideran que siempre tienen un alto porcentaje de ventas, por el hecho de ser productores, lo que les permite tener clientes fieles con lo que suelen trabajar bajo pedidos.

Tabla 3. Tipo de empresa

\begin{tabular}{|l|l|}
\hline PREGUNTA & PERSONAS \\
\hline Mayorista & 15 \\
\hline Minorista & 40 \\
\hline TOTAL & 55 \\
\hline
\end{tabular}




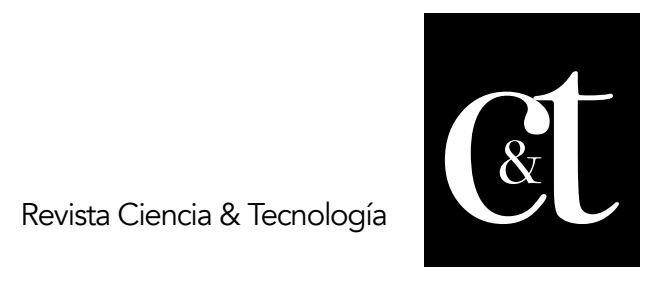

No. 17, 31 de enero de 2018

ISSN impreso: 1390 - 6321

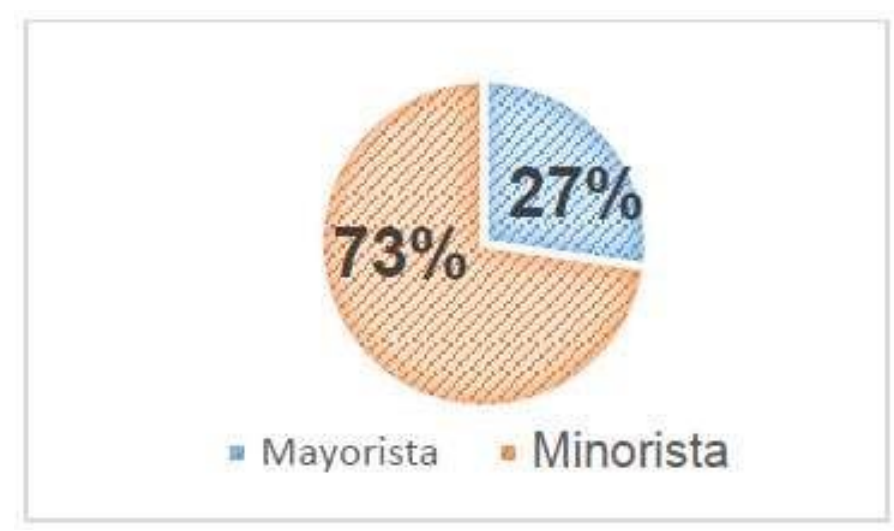

Gráfico 3. Tipo de empresa

Las PYMES del sector se consideran minoristas, por el hecho de que la producción se realiza de forma artesanal y no sobrepasan a los 15 empleados los cuales son oriundos del mismo sector.

\section{Tabla 4. Calidad de Productos}

\begin{tabular}{|l|l|}
\hline Pregunta & Personas \\
\hline Alto & 37 \\
\hline Medio & 13 \\
\hline Bajo & 0
\end{tabular}

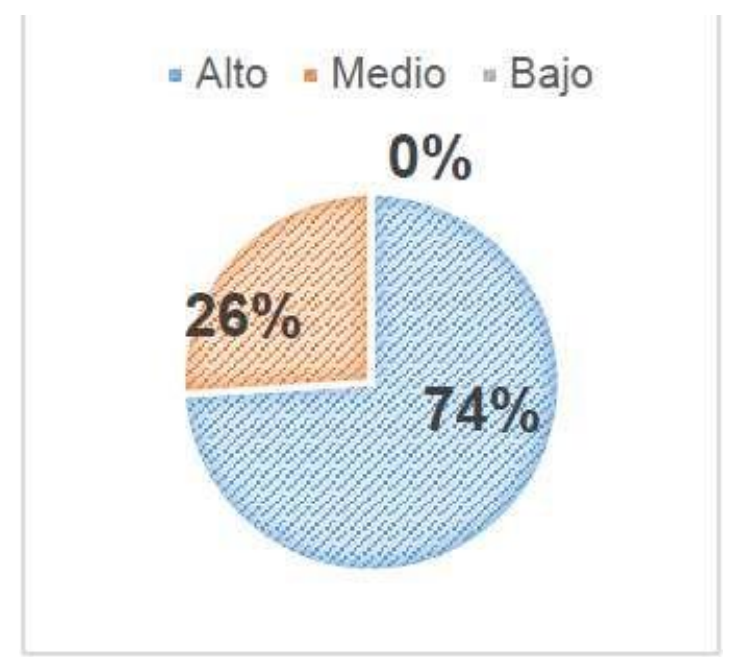

Gráfico 4. Calidad de Productos 


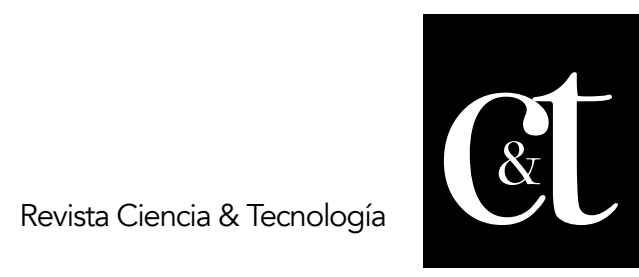

No. 17, 31 de enero de 2018

ISSN impreso: 1390 - 6321

La mayor parte de empresas del sector consideran que sus productos son de alta calidad, lo que influye su venta y comercialización.

Tabla 5. Promoción del producto

\begin{tabular}{|l|l|}
\hline Pregunta & Personas \\
\hline SI & 16 \\
\hline NO & 34 \\
\hline
\end{tabular}

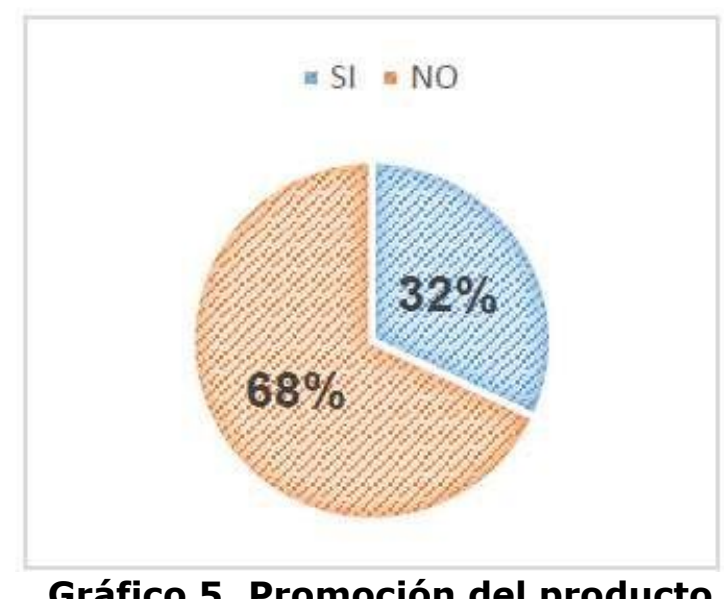

Gráfico 5. Promoción del producto

La mayor parte de las PYMES no utilizan ningún tipo de promoción, ni promociones para dar a conocer sus productos en el sector. 
Tabla 6. Adaptación a cambios

\begin{tabular}{|l|l|}
\hline Pregunta & Personas \\
\hline Siempre & 23 \\
\hline A veces & 20 \\
\hline Nunca & 7 \\
\hline
\end{tabular}

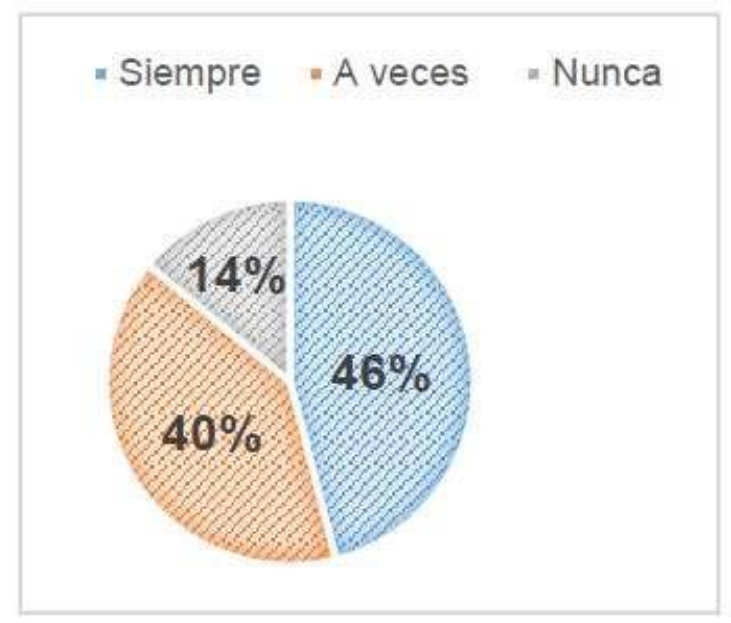

Gráfico 6. Adaptación a cambios

Los propietarios de las PYMES encuestadas han dicho que sus empresas se han adaptado apropiadamente a los distintos cambios ocurridos en nuestro país.

Tabla 7. Calidad de la materia prima utilizada

\begin{tabular}{|l|l|}
\hline Pregunta & Personas \\
\hline Alto & 30 \\
\hline Medio & 20 \\
\hline Bajo & 5 \\
\hline
\end{tabular}




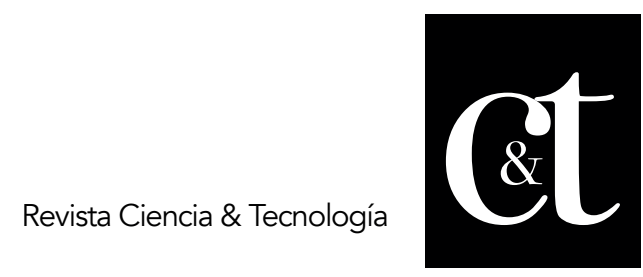

No. 17, 31 de enero de 2018

ISSN impreso: 1390 - 6321

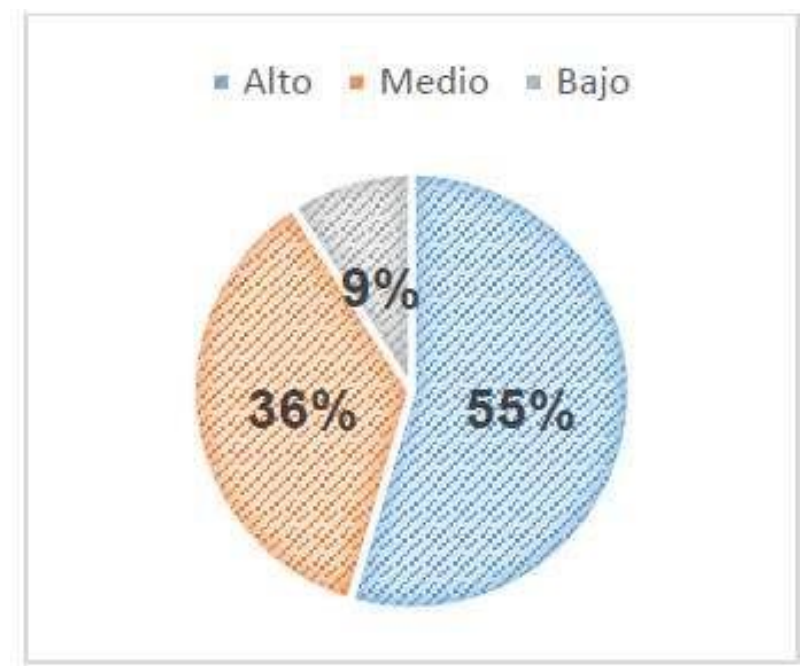

Gráfico 7. Calidad de la materia prima utilizada

La producción de calzado que se realiza en las PYMES de la parroquia de Ambatillo se realiza con materia prima de alta calidad en un $55 \%$ y de mediana calidad en un $36 \%$, es por eso que sus productos tienen una gran demanda en todo el país, en efecto, la mayor parte de la producción es comercializada en la ciudad de Quito y otras ciudades importantes en el Ecuador.

\section{Conclusiones}

En el trabajo realizado con las PYMES del sector Ambatillo se puede llegar a la conclusión de que existe una gran competitividad en el sector y es por eso que sus dueños han visto la forma de adaptarse de la mejor manera para poder incrementar sus ventas.

La mayor parte de las PYMES del sector se considera como empresas minoristas dada las características de su producción que es realizada de forma artesanal y el número de empleados que no es mayor a 15 realizando sus productos con materia prima de alta calidad para así poder lograr confianza en sus clientes y que así ellos sean fieles a sus productos.

Debido a los distintos cambios ocurridos en nuestro país como el terremoto y la crisis en la que nos encontramos las PYMES han tenido que lograr una adopción constante en la producción de sus productos sin disminuir su calidad, brindando así lo mejor a sus clientes.

\section{Referencias bibliográficas}

Abrahamson, H. B. (1990). Administración de empresas medianas y pequeñas. México: Limusa.

Anzola, S. (2015). Administración de pequeñas empresas. México: Mc Graw Hill. 


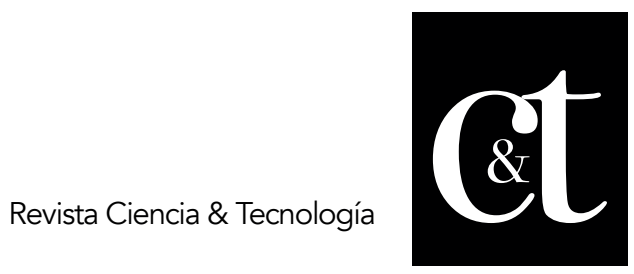

No. 17, 31 de enero de 2018

ISSN impreso: 1390 - 6321

Apps, C., Sterzing, T., Brien, T. O., \& Lake, M. (2017). Lower Limb Joint Stiffness and Muscle Co-contraction Adaptations to. Journal of Electromyography and Kinesiology 31:55-62. https://doi.org/10.1016/j.jelekin.2016.09.003

Area, M. (2013). Realidad de la capacitación y sus necesidades en los gerentes de producción del sector del calzado, cuero y sus manufacturas del Área Metropolitana de Cúcuta (Colombia). AD-minister [online], n.23, pp.151-166.

Davis, A. M., Galna, B., Murphy, A. T., Williams, C. M., \& Haines, T. P. (2016). Gait \& Posture Effect of footwear on minimum foot clearance, heel slippage and spatiotemporal measures of gait in older women. Gait \& Posture, 44, 43-47. https://doi.org/10.1016/j.gaitpost.2015.11.003

Esteban, F., \& Cohen, M. (2013). Sustentabilidad: Un Nuevo Impulso a la Salud Ocupacional. Cienc Trab. vol.15 no.47: 63-66.

Flores, C., Díaz, L., Luz, V., Rodríguez, P. \& Páramo, D. (2015). Evaluación Cuantitativa de la Satisfacción Laboral en Personal Directivo y Operativo de Empresas de Calzado y Cuero Usuarios del IMSS, como Modelo de Atención. Ciencia \& Trabajo Año 17 número 54, septiembre / diciembre 2015: 202-206.

Hernández-Girón, I., Calzado, A., Geleijns, J., Joemai, R. M. S., \& Veldkamp, W. J. $\mathrm{H}$. (2015). Low contrast detectability performance of model observers based on CT phantom images: kVp influence. Physica Médica, 1-10. https://doi.org/10.1016/j.ejmp.2015.04.012.

Irzmanska, E. (2016). Evaluating the comfort of use of protective footwear: Validation of a method based on microclimate parameters and peripheral blood flow. Measurement 77 (2016): 34-39.

Karimi, Z., Allahyari, T., Reza, M., \& Khalkhali, H. (2016). Influence of unstable footwear on lower leg muscle activity, volume change and subjective discomfort during prolonged standing. Applied Ergonomics, 53, 95-102. https://doi.org/10.1016/j.apergo.2015.09.003

Knight, G. (2000). Entrepreneurship and marketing strategy: The SME under globalization. Journal of International Marketing, Vol. 8, No. 2, pp. 12-32.

Luigi, G., \& Antonio, J. (2016). Investigaciones de Historia Económica - Economic History Research The business of fashion in the nineteenth and twentieth centuries. Investigaciones de Historia Económica - Economic History Research, 12(2), 68-75. https://doi.org/10.1016/j.ihe.2016.03.017

Marco, C., \& Merlo, E. (2016). Investigaciones de Historia Económica - Economic History Research Patenting fashion: Salvatore Ferragamo between craftmanship and industry. Investigaciones de Historia Económica - Economic History Research, 12(2), 109-119. https://doi.org/10.1016/j.ihe.2015.07.010.

Mu, M., García-Pinillos, F., \& Soto-Hermoso, V. M. (2016). Acute effects of barefoot running and running requirement on lower-limb kinematics in habitually shod endurance runners. Apunts sport medicine, Vol. 52 Issue 195: 85-91. https://doi.org/10.1016/j.apunts.2016.07.006. 
Ortiz, V.K. \& Caicedo, A.J. (2014). Mezcla óptima de producción desde el enfoque gerencial de la contabilidad del throughput: el caso de una pequeña empresa de calzado. Cuad. Contab, 15 (37): 109-133 / enero-junio 2014. https://doi.org/10.11144/Javeriana.cc15-37.mopd.

Ortiz-Triana, V.K. \& Caicedo-Rolón, Á.J. (2014). Programación óptima de la producción en una pequeña empresa de calzado - en Colombia. Ing. Ind. vol.35 no.2: $114-130$.

Pacheco-Blanco, B., Collado-Ruiz, D., \& Capuz-Rizo, S. (2015). Identification of impacts of stages and materials on life cycle of footwear. Identificación de etapas y materiales de mayor impacto en el ciclo de vida del calzado, DYNA 82(189), 134-141.

Palmer, J. E. R., Montalvo, P. L., \& Pletea, A. M. (2010). Evidencias disponibles sobre las propiedades médicas del calzado tipo Masai type footwear. Atención Primaria, 45(9), 497-498. https://doi.org/10.1016/j.aprim.2013.01.015.

Rengifo, L., Mena, E., Ramírez, G., \& Montoya, A. (2013). Diagnóstico del eslabón curtiembres con base en la matríz Kepner - Tregoe modificada en Pymes del sector cuero, calzado y sus manufacturas del Valle del Cauca. 11th Latin American and Caribbean Conference for Engineering and Technology.

Romero Sigüenza, K. (2015). Análisis de la competitividad del sector del calzado en el Ecuador en el período 2008 - 2013. Tesis de Maestría, Universidad Católica de Santiago de Guayaquil.

Stoney, D. A., Bowen, A. M., \& Stoney, P. L. (2016). Loss and replacement of small particles on the contact surfaces of footwear during successive exposures. Forensic Science International, 269, 78-88. https://doi.org/10.1016/j.forsciint.2016.11.015.

Urriolagoitia, G., \& Coronado, R. (2010). Efecto de la postura y el calzado en la transmisión de vibración en tobillo, rodilla y cadera durante vibración de cuerpo completo. Revista Facultad de Ingeniería Universidad de Antioquia No. 53: 135144.

Whittington, R. (1993). What Is Strategy - And Does It Matter. Routledge: Londres y Nueva York.

Wisbert, E. J. (1969). Enciclopedia Práctica de Management: organización de pequeñas y grandes empresas. España. 